\title{
EDITORIAL
}

\section{NURSES AS THE INDISPUTABLE HEROES OF THE CURRENT PANDEMIC}

\section{Rastislav Mad'ar}

Head of Institute of Epidemiology and Public Health, Faculty of Medicine, University of Ostrava, Czech Repubic Dean of Faculty of Medicine, University of Ostrava, Czech Repubic

Dear colleagues,

For over a year, the world has been fighting a pandemic that has, directly or indirectly, hit every single country and has necessitated unprecedented measures, with hundreds of millions of people globally being quarantined. Expressions like isolation, quarantine, incubation period or reproduction number have become part of everyday conversation. And so have curfew and lockdown, words not used by entire generations in most countries. During this critical period of time, when not only human health and lives but also economies and, in a certain sense, the integrity of civil society are under threat, the nursing profession once again has shown to be of great importance to all of us. Every crisis uncovers the cornerstones that matter most. And it was nurses who became heroes of the pandemic, without whom the fight against coronavirus would not have been successful or even possible. But it has been difficult for them as well, with many of them working under stressful conditions, being justly worried about their own lives or the risk of transmitting the virus to their loved ones or friends. As a result, these strange times have witnessed an interesting phenomenon: professionals normally getting ovations, like athletes or actors, applauded nurses, doctors and other front-line health workers who usually do their job without audiences in near-absolute silence, the only noise being that of equipment, for example in intensive care units.

As a dean of a faculty of medicine, I am proud of our students of nursing and midwifery, but in fact all health science students who have helped during the pandemic. Together with their teachers, they have shown that the so-called third role of universities may be very beneficial to the whole society, an important value added to teaching and research.

Let me thank all of you somehow involved in fighting Covid-19. The battle for human lives is far from being over; after more than a year of hard and tiring work, more challenges are ahead. One of the biggest tasks is the fight against time to achieve herd immunity through vaccination before more dangerous variants of the novel coronavirus spread globally.

I would like to wish all readers of the Central European Journal of Nursing and Midwifery a prosperous year, hoping that reading this issue will be an enriching experience.

Assoc. Prof. Rastislav Mad'ar, MD, Ph.D, MBA. FFTM RCPS (Glasg.)

e-mail: rastislav.madar@osu.cz 